\title{
Teores de cromo ligados aos óxidos de ferro em áreas de descarte de lodo de curtume
}

\author{
Chromium contents linked to iron oxide at areas with tannery sludge disposal
}

\author{
Maurízio Silvera Quadro', Robson Andreazza², Marino José Tedesco3, \\ Clésio Gianelo ${ }^{4}$, Amauri Antunes Barcelos ${ }^{5}$, Leandro Bortolon ${ }^{6}$
}

口-

\begin{abstract}
RESUMO
O descarte de resíduos de lodo de curtume no solo tem sido um problema ambiental devido aos altos teores de cromo. O objetivo deste trabalho foi avaliar a dinâmica dos óxidos de ferro e os teores de cromo ligados a esses minerais em áreas de descarte de resíduos de curtume. Para isso, foram determinados os teores de ferro e cromo extraídos por oxalato de amônio dos óxidos de baixa cristalinidade e por ditionito-citrato-bicarbonato (DCB) dos óxidos cristalinos na fração argila do solo. Esses teores variaram entre 3,5 e $4,1 \mathrm{mg} \mathrm{kg}^{-1}$ nos tratamentos sem aplicação de lodo de curtume e entre 19,9 e 56,8 mg kg ${ }^{-1}$ nos com adição de lodo. Os teores de cromo extraídos dos óxidos cristalinos variaram entre 21,3 e $23,3 \mathrm{mg} \mathrm{kg}^{-1}$ nos tratamentos sem aplicação de lodo e entre 46,4 e 103,0 mg kg nos com uso de lodo de curtume. A aplicação desse material proporcionou um aumento na relação do ferro extraído do óxido de ferro e o ferro extraído com o ditionito $\left(\mathrm{Fe}_{\mathrm{o}} / \mathrm{Fe}_{\mathrm{d}}\right)$ dos óxidos da área de descarte, o que evidencia uma diminuição da cristalinidade dos óxidos. Foi encontrada uma redução na relação do ferro extraído com o ditionito e do cromo extraído com o ditionito $\left(\mathrm{Fe}_{\mathrm{d}} / \mathrm{Cr}\right.$ ), o que mostra a substituição do ferro pelo cromo nos óxidos, evidenciando uma troca isomórfica. Essa alteração pode ser uma alternativa para a retenção de cromo pelo solo com grande força.
\end{abstract}

Palavras-chave: metais pesados; argilominerais; descarte de resíduos.

\begin{abstract}
The tannery sludge disposal in the soil has been a serious environmental problem due to the high chromium concentrations. The aim of this study was to evaluate the iron oxides dynamic and chromium concentrations linked to these minerals in areas of tannery sludge disposal. So, concentration of iron and chromium extracted with ammonium oxalate from oxides with low crystallinity and with dithionite citratebicarbonate from the crystalline oxides in the clay fraction was evaluated. The concentration of chromium extracted from the iron oxides with low crystallinity varied between 3.5 and $4.1 \mathrm{mg} \mathrm{kg-1} \mathrm{in} \mathrm{the} \mathrm{treatments} \mathrm{without} \mathrm{application} \mathrm{of} \mathrm{tannery,}$ and between 19.9 and $56.8 \mathrm{mg} \mathrm{kg-1} \mathrm{in} \mathrm{the} \mathrm{treatments} \mathrm{with} \mathrm{tannery} \mathrm{application.}$ The concentration of chromium extracted from the crystalline oxides varied between 21.3 and $23.3 \mathrm{mg} \mathrm{kg-1}$ in the treatments without application of tannery sludge, and between 46.4 and 103.0 mg kg-1 in the treatments with tannery. The application of tannery promoted an increase in the relation between theiron extracted from theiron oxidesand theiron extracted with dithionite(Feo/Fed)and theoxides from thedisposal area, which is an evidence of the oxide crystallinity decrease. A reduction was found in the relation between the iron extracted with dithionite and the chromium extracted with dithionite (Fed/Crd), what shows the substitution of the iron by chromium in the oxides, emphasizing an isomorphic substitution. This isomorphic substitution can be an alternative to maintain the chromium in the soil with strong retention.
\end{abstract}

Keywords: heavy metals; clay minerals; disposal of waste.

\section{INTRODUÇÃO}

O lodo de curtume é um resíduo gerado durante o processo de transformação de peles em couros. O potencial poluente desses restos é devido à sua elevada carga orgânica, bem como à presença de fenóis, sulfetos e cromo. Além disso, a aplicação desses detritos no solo propicia alterações ambientais que promovem efeitos sobre as enzimas, os organismos e as plantas (PATEL; PATRA, 2014; 2015). Contudo, um

dos maiores problemas ambientais dos lodos de curtume é o cromo, metal pesado que em altas concentrações é tóxico à maioria dos seres vivos, inclusive para os humanos.

O cromo ocorre no lodo de curtume na forma trivalente [Cr (III)], sendo que em condições de baixa acidez esse elemento possui pequena solubilidade (TREBIEN et al., 2011). Devido à sua baixa afinidade por $\mathrm{O}_{2}$, o Cr (III) forma um grande número de complexos, tanto

Doutor em Ciência do Solo. Professor do Centro de Engenharias da Universidade Federal de Pelotas (UFPel) - Pelotas (RS), Brasil. ${ }^{2}$ Pós-Doutor em Ciência do Solo. Professor do Centro de Engenharias da UFPel - Pelotas (RS), Brasil.

${ }^{3}$ Doutor em Ciência do Solo. Professor Titular do Departamento de Solos da Universidade Federal do Rio Grande do Sul (UFRGS) - Porto Alegre (RS), Brasil.

${ }^{4}$ Doutor em Recursos Hídricos. Professor Titular do Departamento de Solos da UFRGS - Porto Alegre (RS), Brasil.

${ }^{5}$ Doutor em Ciência do Solo. Professor do Centro de Engenharias da UFPel - Pelotas (RS), Brasil.

${ }^{6}$ Doutor em Ciência do Solo. Pesquisador da Empresa Brasileira de Pesquisa Agropecuária (EMBRAPA) - Palmas (TO), Brasil.

Endereço para correspondência: Robson Andreazza - Rua Benjamin Constant, 989 - Centro Histórico - 96010-020 - Pelotas (RS), Brasil - E-mail: robsonandreazza@yahoo.com.br Recebido em: 12/O2/16 - Aceito em: 19/12/16 - Reg. ABES: 159157 
com ligantes orgânicos quanto com inorgânicos (LOSI; AMRHEIN; FRANKERBERGER JR., 1994). Entre os ligantes $\mathrm{OH}^{-}, \mathrm{SO}_{4}^{2-}, \mathrm{NO}_{3}^{-}$e $\mathrm{CO}_{3}^{2-}$ avaliados em concentrações comuns em ambientes naturais, somente $\mathrm{OH}^{-}$completa significativamente o $\mathrm{Cr}$.

Desse modo, a formação de hidróxidos insolúveis é preconizada como reação predominante do $\mathrm{Cr}$ (III) no solo, com base nas quantidades de cromo extraídas em análises sequenciais e pelo baixo produto de solubilidade do $\mathrm{Cr}(\mathrm{OH})_{3}\left(\mathrm{Kps}=2,9 \times 10^{-29}\right)$ (JAMES; BARTLETT, 1983).

A matéria orgânica é um dos principais inibidores de cristalização dos óxidos de ferro. Além disso, os grupos funcionais da matéria orgânica bloqueiam o crescimento dos cristais devido à alta afinidade que possuem com sítios superficiais desses minerais (SCHWERTMANN, 1966). A incorporação de lodo de curtume ao solo aumenta os teores de matéria orgânica, principalmente por conter ácidos orgânicos de alto peso molecular e substâncias húmicas estáveis (TREBIEN et al., 2011), provocando alterações no pedoambiente, no qual os óxidos de ferro são estáveis.

As alterações nas condições pedoambientais em que os óxidos de ferro foram formados podem determinar a sua instabilidade e dissolução, bem como a reprecipitação (neoformação) de novos tipos na busca de um novo equilíbrio (COELHO et al., 2008). Com isso, pode acontecer a dissolução e consequente recristalização dos óxidos de ferro; posteriormente, ocorre a substituição isomórfica de cátions nos octaedros estruturais. Nesse sentido, essa substituição é feita pelo alumínio em cristais de hematita e goethita (SCHULZE; SCHWERTMANN, 1984), e o aumento dessa troca está relacionado a pedoambientes altamente intemperizados, nos quais a alta atividade do alumínio é determinada pelo baixo $\mathrm{pH}$ ou menor concentração de silício passível de combinar com o alumínio na solução do solo (SCHWERTMANN; KÄMPF, 1985).

Essa dinâmica dos óxidos de ferro, determinada por processos de dissolução/neoformação, tem permitido a interpretação de mudanças pedoambientais ao longo da gênese dos solos. Assim, o objetivo deste trabalho foi avaliar a dinâmica dos óxidos de ferro e os teores de cromo ligados aos óxidos em uma área de descarte de resíduos de curtume.

\section{MATERIAL E MÉTODOS}

Foi iniciado em 1996, sob campo nativo, um experimento de campo de longa duração na Estação Experimental Agronômica da Universidade Federal do Rio Grande do Sul (EEA/UFRGS), no município de Eldorado do Sul (RS), região fisiográfica da Depressão Central, nas coordenadas geográficas $30^{\circ} 05^{\prime} 76^{\prime \prime} \mathrm{S}$ de latitude e $51^{\circ} 40^{\prime} 67^{\prime \prime} \mathrm{W}$ de longitude. Na área do experimento foi aplicado lodo de curtume nos anos de 1996 e 2000. As características físico-químicas do solo no momento da instalação do experimento eram:
- $\quad$ argila $250,0 \mathrm{~g} \mathrm{~kg}^{-1}$;

- matéria orgânica $25,0 \mathrm{~g} \mathrm{~kg}^{-1}$;

- nitrogênio total $1,12 \mathrm{~g} \mathrm{~kg}^{-1}$;

- $\mathrm{pH}$ em água 4,9;

- $\quad$ índice SMP 5,9; P disponível 3,0 $\mathrm{mg} \mathrm{dm}^{-3}$;

- Al trocável 6,0 $\mathrm{mmol}_{\mathrm{c}} \mathrm{dm}^{-3}$;

- CTC 53,0 $\mathrm{mmol}_{\mathrm{c}} \mathrm{dm}^{-3}$;

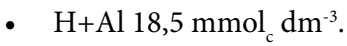

A área experimental estava sob campo nativo antes do início do teste, em 1996. No ano agrícola de 1996/1997, foram cultivados milho e soja, tendo sido as avaliações das culturas e do solo apresentadas por Ferreira et al. (2003). A área permaneceu em pousio até 1999. No ano agrícola de 1999/2000, os resíduos foram novamente aplicados, tendo sido avaliada a disponibilidade dos nutrientes provenientes dos resíduos para as culturas do milho e da soja no verão e do trigo no inverno, sendo os resultados das avaliações das culturas e do solo apresentados por Kray et al. (2008).

As características químicas dos resíduos são apresentadas na Tabela 1. Os tratamentos utilizados foram: $\mathrm{T} 1$ = Controle; $\mathrm{T} 2$ = NPK+calcário; $\mathrm{T} 3=\mathrm{L} 1+\mathrm{PK} ; \mathrm{T} 4=\mathrm{L} 2+\mathrm{PK} ; \mathrm{e} \mathrm{T} 5=\mathrm{L} 1+\mathrm{PK}+\mathrm{Cr}$ mineral. $\mathrm{L} 1$ representa a dose de lodo de curtume aplicado em 1996, e L2, a aplicada em 2000 para atingir pH 6,0 (KRAY et al., 2008; FERREIRA et al., 2003). As duas aplicações de fertilizantes e corretivos da acidez do solo foram feitas conforme as recomendações da Comissão de Química e Fertilidade do Solo do Rio Grande do Sul e de Santa Catarina - CQFS-RS/SC (SBCS/NRS, 2004). As quantidades de lodo de curtume foram aplicadas com base nos resultados de análise de solo e na necessidade de corretivo da acidez do solo (nas duas ocasiões). No T3, foi aplicada a quantidade necessária para elevar o $\mathrm{pH}$ do solo a 6,0; no T4, foi aplicado

Tabela 1 - Características físico-químicas dos resíduos utilizados na aplicação e na reaplicação dos tratamentos.

\begin{tabular}{l|c|c}
\multirow{2}{*}{ Parâmetros } & \multicolumn{2}{|c}{ Lodo de curtume } \\
\cline { 2 - 3 } Teor de sólidos $\left(\mathrm{g} \mathrm{kg}^{-1}\right)$ & $\mathrm{I}^{*}$ & $\mathrm{I}^{* *}$ \\
\hline pH em água & 390,00 & 300,00 \\
\hline Carbono orgânico $\left(\mathrm{g} \mathrm{kg}^{-1}\right)$ & 7,80 & 7,70 \\
\hline Nitrogênio total $\left(\mathrm{g} \mathrm{kg}^{-1}\right)$ & 65,10 & 203,80 \\
\hline $\mathrm{NH}_{4}{ }^{+}\left(\mathrm{mg} \mathrm{L}^{-1}\right)$ & 9,80 & 32,50 \\
\hline $\mathrm{NO}_{3}{ }^{+} \mathrm{NO}_{2}{ }^{-}\left(\mathrm{mg} \mathrm{L}^{-1}\right)$ & 4,20 & 5,10 \\
\hline Fósforo total $\left(\mathrm{g} \mathrm{kg}^{-1}\right)$ & 6,82 & 7,20 \\
\hline Potássio total $\left(\mathrm{g} \mathrm{kg}^{-1}\right)$ & 2,00 & 2,50 \\
\hline Cálcio total $\left(\mathrm{g} \mathrm{kg}^{-1}\right)$ & 0,10 & 0,12 \\
\hline Magnésio total $\left(\mathrm{g} \mathrm{kg}^{-1}\right)$ & 20,00 & 22,00 \\
\hline Enxofre total $\left(\mathrm{g} \mathrm{kg}^{-1}\right)$ & 0,24 & 3,50 \\
\hline Sódio total $\left(\mathrm{g} \mathrm{kg}^{-1}\right)$ & 13,00 & 15,00 \\
\hline Cromo total $\left(\mathrm{g} \mathrm{kg}^{-1}\right)$ & 8,40 & 8,10 \\
\hline Poder de neutralização (\%) & 8,10 & 22,20 \\
\hline
\end{tabular}

"1a aplicação realizada em 12/1996 por Ferreira et al. (2003).

"2a aplicação realizada em 01/2000 por Kray et al. (2008). 
o dobro da dose para elevar o $\mathrm{pH}$ a 6,0; e no T5 foi aplicada a quantidade para elevar o $\mathrm{pH}$ do solo a 6,0 mais $500 \mathrm{~kg}$ de cromo mineral. As quantidades de corretivos da acidez do solo (lodo de curtume e calcário) e de cromo acumuladas em cada parcela são demonstradas na Tabela 2. Foi utilizado o delineamento experimental em blocos ao acaso, com quatro repetições, sendo as unidades experimentais subdivididas para o plantio.

As amostras de solos foram coletadas em agosto de 2005 na camada de 0 a $20 \mathrm{~cm}$ do solo das parcelas de cada tratamento. A amostragem foi realizada com pá de corte, em cinco subamostras por parcela. Posteriormente, o solo coletado foi destorroado e tamisado em peneira de $2 \mathrm{~mm}$ de diâmetro para caracterização química (Tabela 3 ) (TEDESCO et al., 1995).

Os óxidos de ferro pedogênicos $\left(\mathrm{Fe}_{\mathrm{d}}\right)$ foram extraídos por ditionito-citrato-bicarbonato (DCB) a $80^{\circ} \mathrm{C}$ (MEHRA; JACKSON, 1960), em duas extrações sucessivas, sendo que no mesmo extrato foram determinados os teores de cromo ligados aos óxidos de ferro pedogênico.

Tabela 2 - Tratamentos e doses de materiais aplicados em 12/1996 (1a aplicação) e em 01/2000 ( $2^{\mathrm{a}}$ aplicação) em cada tratamento e caracterização química.

\begin{tabular}{l|c|c|c}
\multirow{2}{*}{ Tratamentos } & $1^{\mathrm{a}}$ aplicação & $2^{\mathrm{a}}$ aplicação & Cromo \\
\cline { 2 - 4 } T1 - Controle & \multicolumn{2}{|c}{$\mathrm{t} \mathrm{ha}^{1}$} & $\mathrm{~kg} \mathrm{ha}^{-1}$ \\
\hline T2 - NPK+calcário & 3,4 calcário & 6,3 calcário & - \\
\hline T3 - L1+PK & 21,25 lodo & 22,4 lodo & 671 \\
\hline T4 - L2+PK & 42,50 lodo & 44,80 lodo & 1.342 \\
\hline & 21,25 lodo & 22,4 lodo & \\
\hline T5 - $\mathrm{Cr}_{\text {min }}+\mathrm{L} 1+P K$ & $0,125 \mathrm{Cr}_{\text {min }}$ & $0,500 \mathrm{Cr}_{\text {min }}$ & 1.296 \\
\hline
\end{tabular}

Os óxidos de ferro de baixa cristalinidade $\left(\mathrm{Fe}_{\mathrm{o}}\right)$ foram extraídos com solução de oxalato de amônio $\left(0,2 \mathrm{M}\left(\mathrm{NH}_{4}\right)_{2} \mathrm{C}_{2} \mathrm{O}_{4} \cdot \mathrm{H}_{2} \mathrm{O}\right)$ a $\mathrm{pH}$ 3 , no escuro, sendo que no mesmo extrato foram determinados os teores de cromo ligados aos óxidos de ferro de baixa cristalinidade (SCHWERTMANN, 1964). Os teores totais de ferro e cromo foram determinados por extração nítrico-perclórica na fração argila e analisados por espectrofotometria de absorção atômica (TEDESCO et al., 1995). Os resultados obtidos foram submetidos à análise da variância e, quando significativos, as médias foram comparadas pelo teste de Tukey com $5 \%$ de probabilidade de erro.

\section{RESULTADOS E DISCUSSÃO}

As aplicações sucessivas de lodo de curtume causaram aumentos significativos nos teores de cromo extraídos com oxalato de amônio (Tabela 4). Os maiores teores de cromo foram encontrados no tratamento com adição deste elemento na forma mineral (T5). Não foram encontradas diferenças significativas entre os tratamentos com aplicação de lodo de curtume (T3 e T4), embora haja uma concentração maior de cromo no T4, pela maior adição do mesmo elemento. No tratamento T5, o aumento dos teores de cromo pode ser atribuído à maior disponibilidade dos sais de cromo adicionados. Esses sais em solução tendem a hidrolizar mais facilmente do que o material orgânico, podendo ser precipitados nos óxidos de ferro e manganês de baixa cristalinidade (COELHO et al., 2008).

O descarte de lodo de curtume no solo causou aumentos significativos nos teores de cromo extraídos com DCB (Tabela 4). Já os teores de cromo encontrados no extrato de $\mathrm{DCB}\left(\mathrm{Fe}_{\mathrm{d}}\right)$ foram, em média,

Tabela 3 - Caracterização química do solo na camada de $0-20 \mathrm{~cm}$.

\begin{tabular}{|c|c|c|c|c|c|c|c|c|}
\hline \multirow{2}{*}{ Tratamentos } & \multirow{2}{*}{$\mathrm{pH}$} & $\mathrm{N}$ & $\mathrm{P}$ & $\mathrm{K}$ & \multirow{2}{*}{$\frac{\mathrm{M} . \mathrm{O}}{\mathrm{g} \mathrm{kg}^{-1}}$} & $\mathrm{Al}$ & $\mathrm{Ca}$ & $\mathrm{Mc}$ \\
\hline & & $\mathrm{g} \mathrm{kg}^{-1}$ & & & & \multicolumn{3}{|c|}{$\mathrm{cmol}_{\mathrm{c}} \mathrm{dm}^{-3}$} \\
\hline T1- Controle & 4,8 & 0,9 & 1,8 & 118 & 24,5 & 0,8 & 1,9 & 1,1 \\
\hline T2 - NPK+calcário & 6,4 & 1,0 & 3,9 & 125 & 25,3 & 0,0 & 4,8 & 2,3 \\
\hline T3 - L1+PK & 6,1 & 1,1 & 5,2 & 120 & 27,1 & 0,0 & 5,1 & 1,4 \\
\hline T4 - L2+PK & 6,6 & 1,2 & 7,8 & 130 & 27,6 & 0,0 & 6,4 & 1,7 \\
\hline $\mathrm{T} 5-\mathrm{Cr}_{\min }+\mathrm{L} 1+\mathrm{PK}$ & 5,9 & 1,2 & 5,9 & 154 & 29,3 & 0,0 & 4,8 & 1,1 \\
\hline
\end{tabular}

Tabela 4 - Teores de ferro e de cromo determinados nos extratos de oxalato de amônio ( $\mathrm{Fe}_{\mathrm{o}}$ ), ditionito-citrato-bicarbonato (Fe $\mathrm{f}_{\mathrm{d}}$ e ácidos nítrico+perclórico ( $\mathrm{Cr}$ "total") da fração argila da camada de $0-20 \mathrm{~cm}$.

\begin{tabular}{|c|c|c|c|c|c|c|}
\hline & \multicolumn{3}{|c|}{ Ferro } & \multicolumn{3}{|c|}{ Cromo } \\
\hline & $\mathrm{Fe}_{\mathrm{o}}$ & $\mathrm{Fe}_{\mathrm{d}}$ & "Total" & $\mathrm{Fe}_{0}$ & $\mathrm{Fe}_{\mathrm{d}}$ & "Total" \\
\hline & \multicolumn{3}{|c|}{$\%$} & \multicolumn{3}{|c|}{$\mathrm{mg} \mathrm{kg}^{-1}$} \\
\hline T1-Controle & $0,19 b^{*}$ & $3,11 a$ & $3,30^{\text {ns }}$ & $13,5 b$ & $23,3 \mathrm{c}$ & $43,4 \mathrm{c}$ \\
\hline T2 - NPK+calcário & $0,43 \mathrm{~b}$ & 3,61a & 3,84 & $14,1 \mathrm{~b}$ & $21,3 \mathrm{c}$ & $45,3 \mathrm{c}$ \\
\hline T3 - L1+PK & 0,73 a & $3,28 a$ & 3,82 & $19,9 \mathrm{~b}$ & $46,4 \mathrm{~b}$ & $216,8 \mathrm{~b}$ \\
\hline $\mathrm{T} 4$ - L2+PK & 0,98 a & $3,15 a$ & 4,33 & $27,5 \mathrm{~b}$ & $54,9 \mathrm{~b}$ & $233,3 a b$ \\
\hline $\mathrm{T} 5-\mathrm{Cr}_{\min }+\mathrm{L} 1+\mathrm{PK}$ & 0,79 a & $3,25 a$ & 3,48 & $56,8 \mathrm{a}$ & $103,0 \mathrm{a}$ & $408,1 \mathrm{a}$ \\
\hline CV (\%) & 21 & 12 & 5 & 1,0 & 1,5 & 9,0 \\
\hline
\end{tabular}

*Médias seguidas pela mesma letra na coluna não diferem entre si pelo teste de Tukey com 5\% de probabilidade de erro; ns: não significativo. 
de 22,5 $\mathrm{mg} \mathrm{kg}^{-1}$ nos tratamentos sem aplicação de resíduos (T1 e T2). Contudo, nos tratamentos com aplicação desses resíduos contendo cromo ocorreram aumentos de 1,06; 1,44 e 3,57 vezes, nos tratamentos T3, T4 e T5, respectivamente. Além disso, o tratamento com maior teor de cromo extraído com o DCB $\left(\mathrm{Fe}_{\mathrm{d}}\right)$ foi o T5. Não houve diferença significativa entre os teores de cromo extraídos desta fração $\left(\mathrm{Fe}_{\mathrm{d}}\right)$ entre os tratamentos com aplicação de lodo de curtume (T3 e T4). Alcântara e Camargo (2001) encontraram maiores teores de cromo adsorvidos em solos com maior teor de argila, óxidos de ferro, alumínio e manganês.

A relação do cromo extraído com o ditionito e do cromo extraído com o ditionito $\left(\mathrm{Cr}_{\mathrm{d}} / \mathrm{Cr}_{\mathrm{t}}\right)$ encontrada foi de 0,47 a 0,53 nos tratamentos sem aplicação de lodo de curtume. Isso demonstra que, nos procedimentos sem aplicação de resíduos, metade desse elemento está ligada aos óxidos de alta cristalinidade. Naqueles com aplicação de lodo foram encontradas relações $\mathrm{Cr}_{\mathrm{d}} / \mathrm{Cr}_{\mathrm{t}}$ que variaram de 0,21 a 0,25 , mostrando que, mesmo sendo feita substituição do ferro pelo cromo nos óxidos de ferro, o grande responsável pela imobilização do cromo adicionado é a precipitação de materiais insolúveis como $\mathrm{Cr}(\mathrm{OH})_{3} \mathrm{e} / \mathrm{ou} \mathrm{Cr}_{\mathrm{x}} \mathrm{Fe}_{\mathrm{x}-1}(\mathrm{OH})_{3}$. Segundo Pantsar-Kallio, Reinikainen e Oksanen (2001), um dos principais mecanismos de controle do cromo no solo é constituído pela formação de precipitados, como $\mathrm{Cr}(\mathrm{OH})_{3} \mathrm{e} \mathrm{Cr}_{\mathrm{x}} \mathrm{Fe}_{1-\mathrm{x}}(\mathrm{OH})_{3}$, e ou adsorvido às superfícies carregadas.

Aplicações anteriores de lodo de curtume (de longo prazo) ao solo provocaram aumentos na razão $\mathrm{Fe}_{\mathrm{o}} / \mathrm{Fe}_{\mathrm{d}}$ (Figura 1), correspondente à diminuição da cristalinidade dos óxidos de ferro pedogênicos (MOTTA; KÄMPF, 1992). A diminuição da cristalinidade dos óxidos de ferro em áreas de descarte de resíduos de curtume pode ser decorrente do aporte de grandes quantidades de matéria orgânica (TREBIEN et al., 2011).

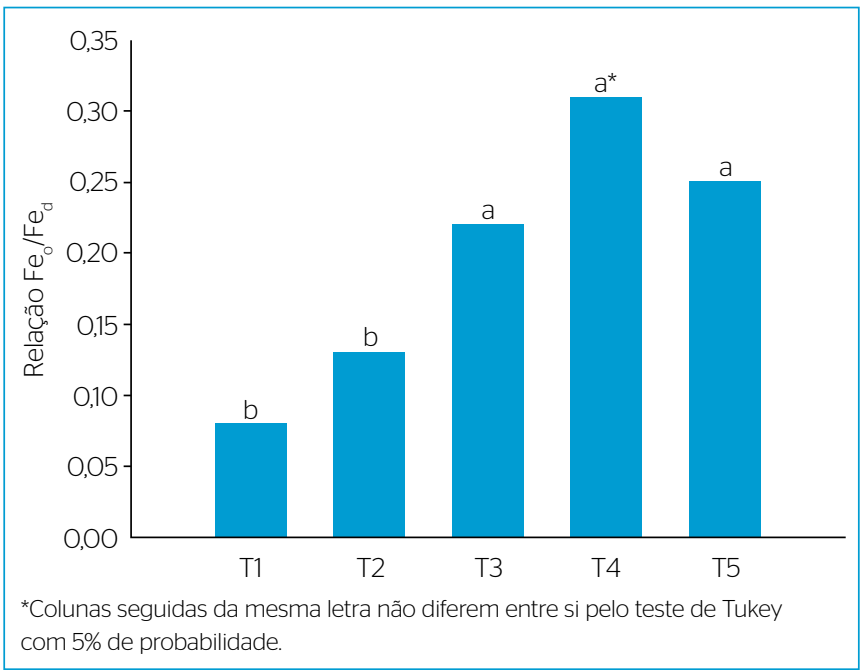

Figura 1 - Razão $\mathrm{Fe}_{\mathrm{o}} / \mathrm{Fe}_{\mathrm{d}}$ na fração argila da camada superficial do solo nos diferentes tratamentos: $\mathrm{T} 1$ = Controle; $\mathrm{T} 2$ = NPK+calcário; $\mathrm{T} 3$ = L1+PK; $\mathrm{T} 4$ = L2+PK; e T5 = L1+PK+Cr mineral.
A substituição do $\mathrm{Fe}^{+3}$ por $\mathrm{Cr}^{+3}$ nos tratamentos sem aplicação de lodo de curtume foi de 0,0006 e $0,0008 \mathrm{~mol} \mathrm{~mol}^{-1}$ para os tratamentos T1 e T2, respectivamente. Já nos tratamentos com aplicação de lodo de curtume a substituição do $\mathrm{Fe}^{+3}$ por $\mathrm{Cr}^{+3}$ foi de 0,0015 e $0,0018 \mathrm{~mol}$ mol $^{-1}$ para os tratamentos T3 e T4, respectivamente. A substituição de $\mathrm{Fe}^{+3}$ por $\mathrm{Cr}^{+3}$ no tratamento com adição de lodo de curtume acrescido de cromo mineral (T5) foi de 0,003 mol mol-1 . Inda Junior e Kämpf (2003) encontraram para goethitas uma amplitude de substituição isomórfica do $\mathrm{Fe}^{3+}$ por $\mathrm{Al}^{3+}$ entre 0,01 e $0,37 \mathrm{~mol} \mathrm{~mol}^{-1}$; nas hematitas, a amplitude foi entre 0,08 e $0,14 \mathrm{~mol} \mathrm{~mol}^{-1}$. Nesse sentido, a aplicação desses resíduos no solo altera o pedoambiente no qual os óxidos de ferro são estáveis, condicionando não só a formação de diferentes tipos de óxidos de ferro, mas também variações mineralógicas dentro de um mesmo mineral (INDA JUNIOR, 2002).

Além disso, ambientalmente, a substituição isomórfica de $\mathrm{Fe}^{+3}$ por $\mathrm{Cr}^{+3}$ pode significar um importante pool para reter o excesso de cromo quando adicionado no solo. Isso é devido a características de toxidez dos metais pesados como o cromo; se houver excesso de metal adicionado no solo, pode haver liberação e ou disponibilidade para as plantas e os organismos do solo, em níveis até mesmo tóxicos, se não houver retenção desses metais pelo solo. Reforçando essa teoria, a substituição isomórfica desse metal pode ser de difícil liberação do mesmo à solução do solo, aumentando o potencial de retenção do cromo pelo solo e indisponibilizando ao ambiente. Diversos estudos indicam que há outros fatores, como estado de óxido-redução, concentração de metal e tipo de sistema de irrigação, que podem afetar a disponibilidade e a toxicidade de cromo para plantas e outros organismos (XIAO et al., 2015).

\section{CONCLUSÕES}

A aplicação de lodo de curtume provocou alterações na dinâmica dos óxidos de ferro, aumentando a relação $\mathrm{Fe}_{\mathrm{o}} / \mathrm{Fe}_{\mathrm{d}}$. Além disso, a relação $\mathrm{Fe}_{\mathrm{o}} / \mathrm{Cr}_{\mathrm{o}}$ e $\mathrm{Fe}_{\mathrm{d}} / \mathrm{Cr}_{\mathrm{d}}$ diminuiu, mostrando uma substituição do ferro pelo cromo nos óxidos. Essa alteração isomórfica pode ser uma alternativa para a retenção de cromo pelo solo com alta força de fixação. Isso demonstra que a aplicação de lodo em um solo afeta a sua estrutura com o tempo; contudo, tal efeito é benéfico na retenção do cromo livre, diminuindo sua disponibilidade ao ambiente e a consequente contaminação ambiental de lugares como águas e/ou solos.

\section{AGRADECIMENTOS}

À Coordenação de Aperfeiçoamento de Pessoal de Nível Superior (CAPES) e ao Conselho Nacional de Desenvolvimento Científico e Tecnológico ( $\mathrm{CNPq})$, pelas bolsas e pelo financiamento do Projeto. 


\section{REFERÊNCIAS}

ALCÂNTARA, M.A.K.; CAMARGO, O.A. (2001) Isotermas de adsorção de freundlich para o crômio (III) em latossolos. Scientia Agricola, v. 58 , p. $567-572$.

COELHO, F.S.; ARDISSON, J.D.; MOURA, F.C.C.; LAGO, R.M.; MURAD, E.; FABRIS, J.D. (2008) Potential application of highly reactive $\mathrm{Fe}(\mathrm{O}) / \mathrm{Fe} 3 \mathrm{O} 4$ composites for the reduction of $\mathrm{Cr}(\mathrm{VI})$ environmental contaminants. Chemosphere, v. 71, p. 90-96.

FERREIRA, A.S.; CAMARGO, F.A.O.; TEDESCO, M.J.; BISSANI, C.A. (2003) Alterações de atributos químicos e biológicos de solo e rendimento de milho e soja pela utilização de resíduos de curtume e carbonífero. Revista Brasileira de Ciência do Solo, v. 27, p. 755-763.

INDA JUNIOR, A.V. (2002) Caracterização de goethita e hematita em solos poligenéticos. Tese (Doutorado) - Programa de PósGraduação em Ciência do Solo, Faculdade de Agronomia, Universidade Federal do Rio Grande do Sul, Porto Alegre.

INDA JUNIOR, A.V.; KÄMPF, N. (2003) Avaliação de procedimentos de extração dos óxidos de ferro pedogênicos com ditionitocitrato-bicarbonato de sódio. Revista Brasileira de Ciência do Solo, v. 27, p. 1139-1147.

JAMES, B.R.; BARTLETT, R.J. (1983) Behavior of chromium in soils. V. Fate of organically complexed $\mathrm{Cr}(\mathrm{III})$ added to soil. Journal of Environmental Quality, v. 12, p. 169-172.

KRAY, C.H.; TEDESCO, M.J.; BISSANI, C.A.; GIANELLO, C.; SILVA, K.J. (2008) Tannery and coal mining waste disposal on soil. Revista Brasileira de Ciência do Solo, v. 32, p. 2877-2882.

LOSI, M.E.; AMRHEIN, C:; FRANKENBERGER JR. W.T. (1994) Environmental biochemistry of chromium. Reviews of Environmental Contamination and Toxicology, v. 136, p. 91-121.

MEHRA, O.P.; JACKSON, M.L. (1960) Iron oxide removal from soils and clays by a dithionite-citrate system buffered with sodium bicarbonate. Clays and Clay Minerals, Ottawa, v. 7, n. 1, p. 317-327.

MOTTA, P.E.F.; KÄMPF, N. (1992) Iron oxide properties as support to soil morphological features for prediction of moisture regimes in Oxisols of central Brazil. Z Pflanzenbau und Bodenkunde, v. 155, p. 385-390.
PANTSAR-KALLIO, M.; REINIKAINEN, S.P.; OKSANEN, M. (2001) Interactions of soil components and their effects on speciation of chromium in soils. Analytica Chimica Acta, v. 439, p. 9-17.

PATEL, A.; PATRA, D.D. (2014) Influence of heavy metal rich tannery sludge on soil enzymes vis-à-vis growth of Tagetes minuta, an essential oil bearing crop. Chemosphere, v. 112, p. 323-332.

PATEL, A.; PATRA, D.D. (2015) Effect of tannery sludge amended soil on glutathione activity of four aromatic crops: Tagetes minuta, Pelargonium graveolens, Ocimum basilicum and Mentha spicata. Ecology Engineering, v. 81, p. 348-352.

SCHULZE, D.G.; SCHWERTMANN, U. (1984) The influence of aluminium on iron oxides: $\mathrm{X}$. Properties of Al-substituted goethites. Clay Minerals, v. 19, p. 521-539.

SCHWERTMANN, U. (1964) Differenzierung der eisenoxide des bodens durch extraktion mitammoniumoxalat-lösung. Z Pflanzenbau und Bodenkunde, v. 105, p. 194-202.

(1966) Inhibitory effect of soil organic matter on the crystallization of amorphous ferric hidroxides. Nature, v. 212, p. 645-646.

SCHWERTMANN, U.; KÄMPF, N. (1985) Properties of goethite and hematite in kaolinitic soils of southern and central Brazil. Soil Science Society of America Journal, v. 139, p. 344-350.

SOCIEDADE BRASILEIRA DE CIÊNCIA DO SOLO/NÚCLEO REGIONAL SUL (SBCS/NRS). (2004). Recomendações de adubação e de calagem para os estados do Rio Grande do Sul e de Santa Catarina. 10. ed. Porto Alegre: SBCS/NRS. 400 p.

TEDESCO, M.J.; GIANELLO, C.; BISSANI, C.A.; BOHNEN, H.; VOLKWEISS, S.J. (1995) Análises de solo, plantas e outros materiais. 2. ed. Porto Alegre: Universidade Federal do Rio Grande do Sul.

TREBIEN, D.O.P.; BORTOLON, L.;TEDESCO, M.J.; BISSANI, C.A.; CAMARGO, F.A.O. (2011) Environmental factors affecting chromium-manganese oxidation-reduction reactions in soil. Pedosphere, v. 21, p. 84-89.

XIAO, W.; YE, X.; YANG, X.; LI, T.; ZHAO, S.; ZHANG, Q. (2O15) Effects of alternating wetting and drying versus continuous flooding on chromium fate in paddy soils. Ecotoxicology Environmental Safety, v. 113, p. $439-445$ 